\title{
A model of distributional handing interaction for a mobile robot
}

\author{
Chao Shi ${ }^{1,2}$ Masahiro Shiomi ${ }^{1}$ Christian Smith $^{3}$ Takayuki Kanda ${ }^{1}$ Hiroshi Ishiguro ${ }^{1,2}$ \\ ${ }^{1}$ ATR IRC Laboratories \\ 2-2-2 Hikaridai Keihanna Science City \\ Kyoto 610-0228 Japan \\ ${ }^{2}$ Osaka University \\ 1-3 Machikaneyama Toyonaka \\ Osaka 565-8531 Japan \\ ${ }^{3}$ Royal Institute of Technology, \\ Stockholm, Sweden
}

shi.chao@irl.sys.es.osaka-u.ac.jp, \{m-shiomi, kanda, ishiguro\}@atr.jp, ccs@kth.se

\begin{abstract}
This paper reports our research on developing a model for a robot distributing flyers to pedestrians. The difficulty is that potential receivers are pedestrians who are not necessarily cooperative; thus, the robot needs to appropriately plan its motion, making it is easy and non-obstructive for potential receivers to receive the flyers. In order to establish the model, we observed human interactions on distributional handing in the real world. We analyzed and evaluated different handing methods that people perform, and established a model for a robot to perform natural handing. The proposed model is implemented into a humanoid robot and is confirmed as effective in a field experiment.
\end{abstract}

Keywords-Human-robot interaction; handing interaction; interaction during walking

\section{INTRODUCTION}

A number of robots have been developed in research projects for serving people in daily public environments. Robots would work as a shopping assistant that approaches a customer to offer assistance in a shopping mall. Robots would talk to visitors and guide directions on the street. Robots would also perform friendly patrolling as a guard for the pedestrians to easily ask for helps in a public space. Other robots work in different environments such as museums, hospitals and homes.

In our daily life, it is very common to see people distribute objects such as flyers to pedestrians. For example, a shop assistant distributing coupons to customers in a shopping mall, a staff distributing pamphlets to visitors in a museum, or a clerk of a barbershop distributing advertisement flyers to pedestrians in front of a crowded station, and so on. It would be appropriate to assume that in the future these "distributing" works would be carried out by robots.

How does a robot take the place of doing such distributing work? Even though this might seem trivial for people, it is not at all trivial for robots. It is difficult for a robot to replicate what humans unconsciously perform. It needs to know every detail of the behavior, such as from which direction it should approach the target person, and where and when it should extend its arm for distributing. Since human beings are doing this unconsciously, intricately describing how exactly we are doing it is not easy.

In this study, we define this distribution interaction as distributional handing and focus in natural Human-robot

This research was sponsored by JST, CREST and JSPS KAKENHI Grant Number 25220004

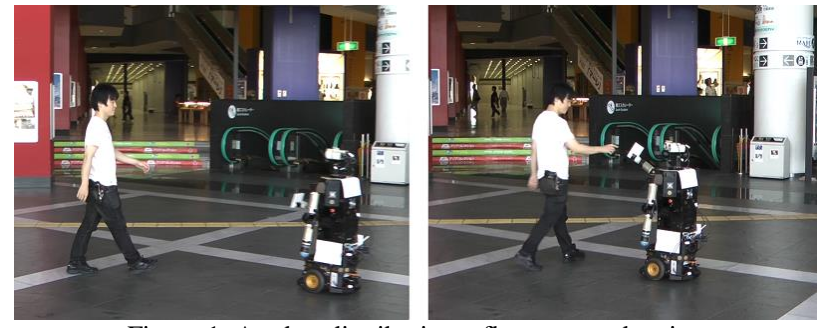

Figure 1. A robot distributing a flyer to a pedestrian

interaction. We first studied distributional handing in humanhuman interaction, and then implemented it into a humanoid robot (Fig. 1).

\section{RELATED WORKS}

\section{A. Studies related to handing behavior}

Handing capability is built on a number of techniques in robotics. For instance, recent progress in mobile manipulation [1-2] are clearly relevant. Some studies have also investigated how to make grasping socially acceptable [3].

Specific to the handing interaction, early studies were devoted to generating natural handing motion, imitating how humans do it [4-6]. Huber et al., showed that a minimum jerk model makes arm motion appear more natural and made the subjects' response times shorter [7]. Cakmak et al., investigated how to design a handing-over motion to convey the moment when the person should receive the object [8] and how to learn a preferable robot configuration for the task [9]. Sisbot et al., showed how to navigate a robot [10] and manipulate objects in the vicinity of humans [11]. Koay et al., presents the results from a Human-Robot Interaction study that investigates the issues of participant's preferences in terms of the robot approaching method and handing over behavior in the context of a robot handing over an object to a seated person [22]. Further, the use of perspective taking for joint manipulation has been addressed [12]. However, most of these researches only focused on the behaviors for handing object to a specified person stopped at a fixed position. While in the case of handing object to walking pedestrians, the necessary knowledge such as how to choose a pedestrian as the handing target person, how to approach the person, at what timing and how should the robot extend its arm to provide the object to the target, would be very different from that in the conventional researches. This knowledge remains unknown. 


\section{B. Studies related to approaching method}

Besides the handing interaction, a few studies have addressed the process of initiating interaction, considering proxemics [13] and inviting behavior [14]. These studies addressed ways for a robot to exhibit intention to initiate interaction, but in these cases the robots were stationary, which makes the situation quite different from distributional handing.

There have been some studies that address how to approach humans. Dautenhahn et al., studied what kind of approaching behavior of a robot was preferred by users, and found that it is better to approach from the side instead of the front when users are seated [15]. While [22] reported that a seated person preferred the robot to hand over him the object by approaching from front side, in contrast with [15]. Shi et al., modeled people's spatial configuration when one person approaches another and implemented it into a robot [16]. Satake et al., proposed a target selection algorithm and path-planning algorithm for proactive approaching, in which a robot aims to meet people from a frontal direction [17]. These studies show that as the situation changes (i.e., the state of the target person, the interaction purpose of the robot, etc.), the approach method of the robot would be different from each other. This implies that we should investigate specifically proper approaching method for distributional handing.

\section{Modeling Distributional Handing}

In some countries, such as Japan, China, etc., clerks from many kinds of shops or institutions often distribute flyers, coupons or pamphlets in many places such as shopping mall, museum or even outdoor. Hence, we decided to model distributional handing based on our observations of the handing behavior conducted by those people who really distribute objects.

As we could easily find in our daily life, people perform distributional handing in various kinds of ways. For example, some givers stand at a certain place with an extending arm, and wait someone in a flow of pedestrian to take it. While some other givers may walk to approach the pedestrians proactively and extend their arms nearby to handing. These differences of behaviors may have different effects on "persuading" the pedestrian to receive the object. It seems that some givers could often make many pedestrians receive the object; while some other givers may fail to give out even one object despite that he tried to handing to every pedestrian passed by. Thus, we consider that performing distributional handing in different ways would make great differences on the distributing efficiency. Our main goal is to find out what is the proper method of distirbutional handing, and then create a model for a humanoid robot.

\section{A. Data Collection}

We conducted our observation in a shopping mall (Fig. 2) in the Osaka bay area, Japan. The area includes a corridor of approx. 70m with 3-6 m width and 4shops alongside, and one big hall of approx. $300 \mathrm{~m}^{2}$ which connects to the corridor. The corridor connects the hall to a train station, and many pedestrians passed through it and the big hall.

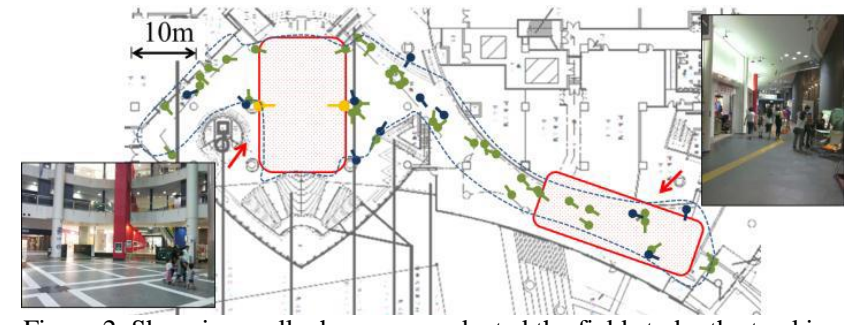

Figure 2. Shopping mall where we conducted the field study: the tracking area is inside the dashed line and the sensor positions are shown as circles. The photos show the corridor and hall areas.

Both the corridor and the hall were covered with our people-tracking infrastructure using 49 3-D range sensors attached on the ceiling (combination of Panasonic D-Imager, ASUS Xtion, and Velodyne HDL-32E), providing an estimation of pedestrians' locations every $33 \mathrm{~ms}$. 16 cameras were attached as well to provide video data.

The data collection of the pedestrians was conducted from 9 am to $8 \mathrm{pm}$ on Sunday every week. By searching our collected data, we picked up 10 givers who worked on distributional handing in the shopping mall and analyzed their behaviors in 40 times for each giver.

\section{B. Analysis of distributional handing}

1) Finding the method of distributional handing:

To find out the proper method of distributional handing, we analyzed and evaluated different methods that the givers performed.

\section{a) Focus of the behaviors in distributional handing :}

Glasauer et al., suggested that for successful joint action the robot must act predictably for the human partner [18]. A study in handing objects to seated person [8] reported that a giver provides a signal by a motion of arm extension, which informs his/her intention of handing an object. Although situations in distributing objects to pedestrians are different from that, we consider that the giver in distributional handing also signals his/her intention. While not studied in [8], it was often reported that gaze and spatial formation plays an important role in signaling to initiate an interaction [16]. Thus, our observation focused on these behaviors as below:

- Gaze: when and how did the giver look at the pedestrian, inferred from face direction

- Approach: when and how did the givers position themselves for the handing

- Arm: when and how did the giver extend his arm to offer the flyer to the pedestrian

To evaluate whether the behaviors of distributional handing done by the givers are good or not, we define a successful ratio for distributional handing. If the target pedestrian received the flyer from the giver, we record the handing as success; if the pedestrian refused to take the flyer, the handing would be recorded as fail.

\section{b) Different types of distributional handing :}

We found that all the givers' gaze behavior were similarly. When a pedestrian has been chosen as the handing target, the givers kept gazing at the pedestrian to keep eye contact until the handing finished. 
In contrast, we found differences in approach and arm behaviors. We noticed that the givers perform distributional handing mainly in four patterns:

- Extend arm first and wait pedestrian: The giver firstly stayed on waiting at a certain place. She noticed a pedestrian and gazed at him (Fig. 3-1-a). The giver then began to extend arm to handing the flyer (fig. 3-1-b). When the giver fully extended her arm and completed the handing motion, the pedestrian is still a little far (Fig, 3-1-c).

- Wait for the pedestrian and extend arm nearby: The giver waited for the pedestrian's coming while kept holding the flyer at the waist-height at the beginning (Fig. 3-2-a). The giver did not start to extend the arm until the pedestrian got very close (Fig. 3-2-b). As shown in Fig. 32 -c, at the time that the giver completed the arm extending motion, distance between the giver and the pedestrian is just proper for the pedestrian to take the flyer instantly.

- Extend arm first and approach pedestrian: The giver waited for the pedestrian's coming, and then noticed the two pedestrians came in pair (Fig. 3-3-a). As the giver chose the pedestrian dressed in black as the handing target, the giver extended the arm holding the flyer in hand and began to approach the pedestrian (Fig. 3-3-b). Instead of just moving to the side of the pedestrian's route and waiting, the giver kept moving towards the pedestrian to approach her. The giver did not stop walking until he reached the place that the pedestrian could easily take the flyer.

- Approach pedestrian and extend arm nearby: The giver noticed a pedestrian came from the right side. She then moved to approach the pedestrian (Fig. 3-4-a). As the distance between the pedestrian and the giver got close, the giver started to extend her arm to distribute the flyer while keeping approaching the pedestrian (fig. 3-4-b). At last, the giver stopped near the pedestrian and completed the arm extending motion at the same time.

We then asked two coders to classify all the 400 trials of

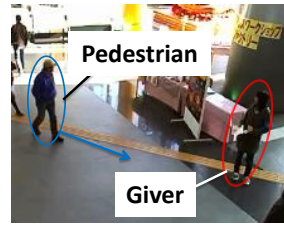

(a)

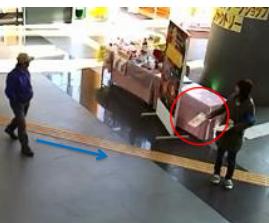

(b)

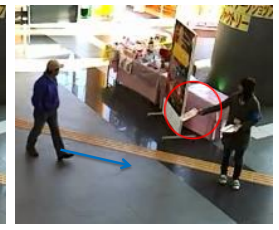

(c)
(1) Extend arm first and wait pedestrian

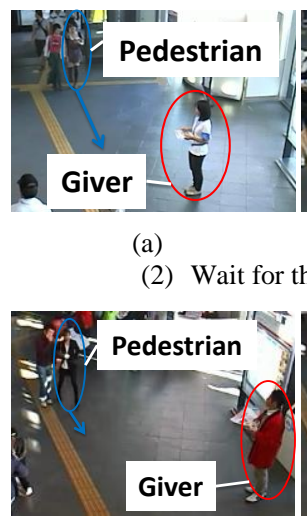

(a)

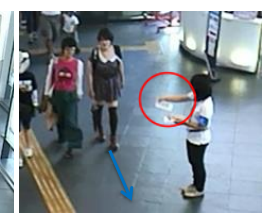

(b)

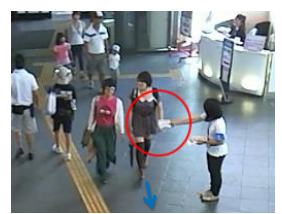

(c)
(2) Wait for the pedestrian and extend arm nearby

(3) Extend arm first and approach pedestrian

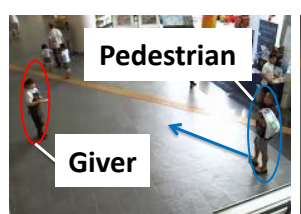

(a)

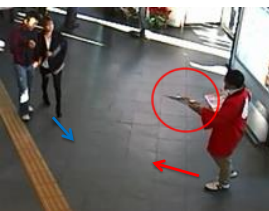

(b)

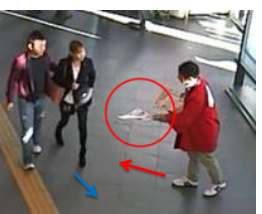

(c)

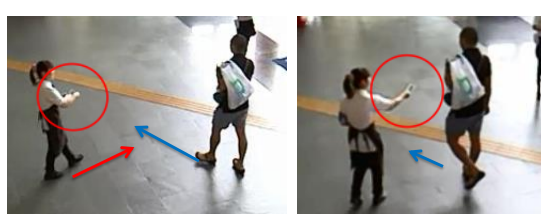

(b) (c)
(4) Approach pedestrian and extend arm nearby

Figure 3. Four types of distributional handing

handing from the 10 givers separately. Cohen's kappa coefficient from the two coder's classifications was 0.873, indicating that their evaluations were highly consistent. After classification, to analyze relationship between the behaviors and the successful ratio, the two coders discussed and reached a consensus in their classification results for the entire coding

TABLE I. Type AND SUCCESSFUl RATIO OF DistRIBUtional HANDING

\begin{tabular}{|c|c|c|c|c|c|}
\hline \multirow{2}{*}{ Giver } & \multicolumn{5}{|c|}{ Type } \\
\cline { 2 - 6 } & $\begin{array}{c}\text { Extend arm first and } \\
\text { wait pedestrian }\end{array}$ & $\begin{array}{c}\text { Wait for the pedestrian } \\
\text { and extend arm nearby }\end{array}$ & $\begin{array}{c}\text { Extend arm first and } \\
\text { approach pedestrian }\end{array}$ & $\begin{array}{c}\text { Approach pedestrian and } \\
\text { extend arm nearby }\end{array}$ & \multirow{2}{*}{ Total } \\
\hline 1 & $0(0 / 0)$ & $40.0 \%(2 / 5)$ & $100.0 \%(2 / 2)$ & $81.2 \%(27 / 33)$ & $77.5 \%(31 / 40)$ \\
\hline 2 & $0(0 / 1)$ & $46.2 \%(6 / 13)$ & $33.3 \%(1 / 3)$ & $78.3 \%(18 / 23)$ & $62.5 \%(25 / 40)$ \\
\hline 3 & $0(0 / 0)$ & $42.9 \%(9 / 21)$ & $0(0 / 0)$ & $78.9 \%(15 / 19)$ & $60.0 \%(24 / 40)$ \\
\hline 4 & $18.2 \%(2 / 11)$ & $38.1 \%(8 / 21)$ & $0(0 / 2)$ & $83.3 \%(5 / 6)$ & $37.5 \%(15 / 40)$ \\
\hline 5 & $42.9 \%(6 / 14)$ & $29.4 \%(5 / 17)$ & $0(0 / 1)$ & $37.5 \%(3 / 8)$ & $35 \%(14 / 40)$ \\
\hline 6 & $21.1 \%(4 / 19)$ & $43.8 \%(7 / 16)$ & $50.0 \%(1 / 2)$ & $33.3 \%(1 / 3)$ & $32.5 \%(13 / 40)$ \\
\hline 7 & $16.7 \%(1 / 6)$ & $36.0 \%(9 / 25)$ & $20.0 \%(1 / 5)$ & $50.0 \%(2 / 4)$ & $32.5 \%(13 / 40)$ \\
\hline 8 & $33.3 \%(3 / 9)$ & $17.4 \%(4 / 23)$ & $0(0 / 2)$ & $66.7 \%(4 / 6)$ & $27.5 \%(11 / 40)$ \\
\hline 9 & $17.6 \%(3 / 17)$ & $30.0 \%(6 / 20)$ & $0(0 / 1)$ & $0(0 / 2)$ & $22.5 \%(9 / 40)$ \\
\hline 10 & $9.1 \%(2 / 22)$ & $13.3 \%(2 / 15)$ & $0(0 / 2)$ & $100.0 \%(1 / 1)$ & $12.5 \%(5 / 40)$ \\
\hline ALL & $21.2 \%(21 / 99)$ & $33.0 \%(58 / 176)$ & $25.0 \%(5 / 20)$ & $72.4 \%(76 / 105)$ & $40.0 \%$ \\
$(160 / 400)$ \\
\hline
\end{tabular}


process. The result was shown in Table 1, which is ordered with total successful ratios. The coding results clearly show that the 10 givers took different style of distributing, and their successful ratios also differ a lot. The important phenomena is that givers achieved highest successful ratio when distributing objects in Approach pedestrian and extend arm nearby type (72.4\%), which is much higher than in the other three methods (Extend arm first and wait pedestrian : $21.2 \%$, Wait pedestrian and extend arm nearby: $33.0 \%$, Extend arm first and approach pedestrian : $25.0 \%$,). The first three givers in Table 1 who achieved high successful ratios distributed flyers very frequently in Approach pedestrian and extend arm nearby method. Therefore, we decided to use Approach and handing closely type behaviors as our model for distributional handing.

\section{2) Detailed Modeling:}

We then modeled the Approach pedestrian and extend arm nearby method and retrieved the parameters.

\section{a) Detailes of the behaviors and timing:}

To create the model, we further analyzed the details of each behavior and the timing.

- Timing: We define the timing that the giver started a behavior as $t_{\text {start, }}$ completed a behavior as $t_{\text {ready }}$. Since gaze behavior could be completed momentarily, we set that $t_{\text {gazestart }}=t_{\text {gazeready }}$. Regarding the timing of $t_{\text {start }}$, we found that the giver always began with gazing the target pedestrian. The givers then perform approaching behavior, and at last perform arm behavior when he/she got closer to the pedestrian. In some cases that the giver realized the pedestrian's coming were very late, the giver started the behaviors simultaneously. For $t_{\text {ready }}$, the givers completed arm behavior just at the same time as he/she completed approaching behavior, i.e., $t_{\text {approachready }}=t_{\text {armready }}$ ( $\max$ error $=0.7 \mathrm{~s}$ ). In summary, we define the following constraints:

$T_{\text {gazestart }}<=t_{\text {armstart }}<=t_{\text {approachstart }}$

$t_{\text {armready }}=t_{\text {approachready }}$

- Details of Approaching: We analyzed the approaching behavior of givers. When handing the object, it is typical for the giver to keep approaching the pedestrian until the pedestrian is close to his/her frontal right/left. While in some cases (12 out of all the 105 trials) that the pedestrian came directly towards the giver's initial position, the giver would avoid from the pedestrian's route and approach the pedestrian from side (fig. 4). Thus, we consider that in distributional handing, it is proper for a giver to approach the pedestrian from frontal left/right side instead of directly front. As shown in Fig. 5, we denote the position of the giver and the pedestrian at $t_{\text {approachready }}$ as $G_{\text {ready }}$ and $P_{\text {ready }}$. We formulate equations for candidates for $G_{\text {ready }}$ when $P_{\text {ready }}$ is estimated as below:

$G_{\text {ready }}\left(i, t_{\text {predict }}\right.$, Side $)= \begin{cases}\text { FrontalLeft }\left(E P_{i}\left(t_{\text {predict }}\right)\right) & (\text { Side }=l e f t) \\ \text { FrontalRight }\left(E P_{i}\left(t_{\text {predict }}\right)\right) & (\text { Side }=\text { right })\end{cases}$

where $E P_{i}(t)$ is the estimated position of pedestrian $i$ at time $t$ given his/her past velocity information, $t_{\text {predict }}$ is the time when the pedestrian can reach $E P_{i}\left(t_{\text {predict }}\right)$, the range of $t_{\text {predict }}$ is searched every $1 \mathrm{sec}$. Side indicates if $G_{\text {ready }}$ is on
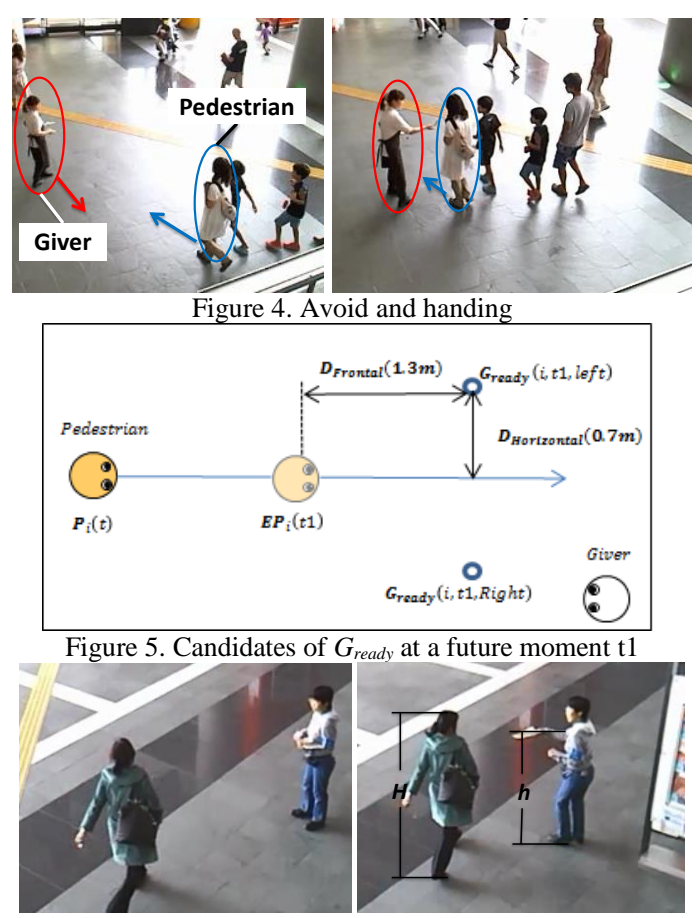

Figure 6. Handing motion observed in the data collection

the left or right side of $E P_{i}\left(t_{\text {predict }}\right)$, FrontalLeft and FrontalRight are the functions to calculate the position of $G_{\text {ready }}$ from $\mathrm{EP}_{\mathrm{i}}(\mathrm{t})$. We decomposed the distance between $P_{\text {ready }}$ and $G_{\text {ready }}$ into frontal (element of the distance in the direction of the pedestrian's motion) and horizontal (in the direction orthogonal to the pedestrian's motion), as shown in figure 5. By adding $D_{\text {frontal }}$ to moving direction and $D_{\text {horizontal }}$ to the orthogonal direction of the moving direction $G_{\text {ready }}$ could be calculated.

- Details of Extending Arm: Previous work [4] suggested that the start and end position of the arm is an important signal to the target person for handing. In the collected data, the giver typically held the flyer just above waist-height from the beginning to $t_{\text {armstart }}$. As getting closer to the pedestrian, the giver started to extend arm at about the same height as the pedestrian's waist-height (fig. 6).We found that the height of the giver's hand is changing according to the pedestrian's height to make the pedestrian be comfortable to receive the flyer. We note that this height is independent of the giver's height. In this paper, we calculate the height of the pedestrian's waist by using a ratio of it to the overall height. As shown in Fig. 6, we set the height of the giver's hand as $h$, the height of the pedestrian as $H$. We set $k$ as the ratio of the height of the giver's hand to the height of the pedestrian. We then formulate a constraint for the height of the hand in the handing motion as follows:

$$
h=k \cdot H
$$

\section{b) Retrieving parameters:}

Since it is difficult to precisely retrieve the parameters from the video data, we asked three givers to act distributional handing in Approach pedestrian and extend arm nearby method in our lab. We collected the data with a motion capture 
system, which tracks the position data in $100 \mathrm{~Hz}$ with error less than $2 \mathrm{~mm}$.

We computed the distance between $P_{\text {ready }}$ and $G_{\text {ready }}$, and found that the average of $\left|G_{\text {ready }}-P_{\text {ready }}\right|$ was $1.5 \mathrm{~m}$ (ranged from $1.2 \mathrm{~m}$ to $1.7 \mathrm{~m}$ ). The average horizontal distance was $0.7 \mathrm{~m}$ (ranged from $0.6 \mathrm{~m}$ to $0.8 \mathrm{~m}$ ) and the frontal distance was 1.3 $\mathrm{m}$ (ranged from $1 \mathrm{~m}$ to $1.5 \mathrm{~m}$ ). From this, we set that the giver tries to keep the horizontal distance $D_{\text {horizontal }}$ at around $0.7 \mathrm{~m}$, frontal distance $D_{\text {frontal }}$ at around $1.3 \mathrm{~m}$.

We used the height of givers' hand and the pedestrians to calculate $k$ (Eq. 2). The average of $k$ is about 0.632 (ranged from 0.617 to 0.676 ). Therefore, we use the ratio $k=0.632$.

\section{IMPLEMENTATION OF DISTRIBUTIONAL HANDING}

From our analysis, we developed a system which controls the robot behavior to satisfy these equations.

Figure 7 shows the system configuration. To cover a large area, we used a person tracking system based on 3-D range sensors which we had explained in Section III-A. There are three processes for the handing interaction. First, the target decider (section IV-C) selects a target person for handing interaction. Once the target is selected, the spatial formation controller controls the robot locomotion to adjust spatial formation (section IV-D), while the gesture controller controls the gaze and the arm behavior of the robot (section IV-E).

\section{A. Robot}

We used the humanoid robot Robovie [19], which is 1.2 $\mathrm{m}$ tall with a $0.3 \mathrm{~m}$ radius and characterized by its human-like body expressions. It has a 3-DOF head and 4-DOF arms with 2-DOF hands. Its locomotion platform is a wheeled Pioneer3 DX. We set it to move at a velocity of $700 \mathrm{~mm} / \mathrm{sec}$ (approx. $2.5 \mathrm{~km} / \mathrm{h}$ ) forward and $45 \mathrm{degree} / \mathrm{sec}$ for rotations. The acceleration of the forward and rotation are $600 \mathrm{~mm} / \mathrm{sec}$ and 30 degree/sec respectively.

To hand out flyers, we attached a mobile printer to the right side of the robot's waist. The robot can print the flyer autonomously before finding a target; we prepared a gesture for the robot to pick it up with its right hand, which is a clip type one (Figure 8).

\section{B. Target Decider}

This module selects a target person for the handing interaction based on position information of pedestrians. For each pedestrian $i$, it tests whether the future moment $t$, at which the pedestrian $i$ will be at $E P_{i}(t)$, can be a good position for handing. In other words, it tests whether $P_{\text {ready }}=E P_{i}(t)$ is satisfied. It calculates the positions where the robot should be for handing, i.e. $G_{\text {ready }}$, and tests whether the set of $\left(i, t, G_{\text {ready }}\right)$ can be realized given the robot's current position. It computes the time required to move from $G_{\text {current }}$ to $G_{\text {ready }}$ with the path shown in Figure 9.

In this computation, it only chooses a plan with enough margin time. There is a parameter TimeToleranceFactor in the following equation, representing the coefficient to make a margin time, which is to cover possible small change of people's moving direction or small underestimation due to noise in sensing. This parameter was empirically set to 1.2 , as

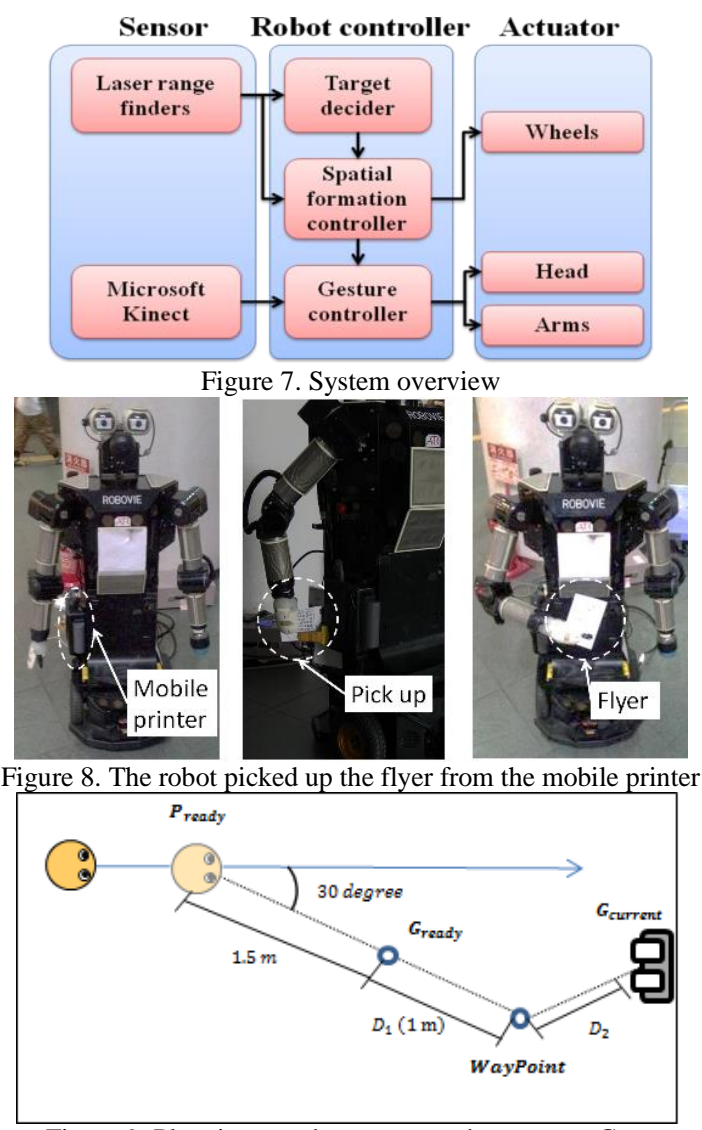

Figure 9. Planning a path to move to the target at $\mathrm{G}_{\text {ready }}$

an enough large value. Overall, the function below tests whether the robot can arrive to $G_{\text {ready }}$ in time:

CanArrive $\left(t, G_{\text {current }}, G_{\text {ready }}\right)=$
$\begin{cases}\text { true } & \left(\text { EstimatedTime }\left(G_{\text {curren }}, G_{\text {ready }}\right) \cdot \text { TimeToleranceFactor }<=t\right) \\ \text { false } & (\text { otherwise })\end{cases}$

where $G_{\text {current }}$ is the current position of the robot, EstimatedTime is a function returning the necessary time given maximum robot velocities - to move the robot from $G_{\text {current }}$ to $G_{\text {ready }}$ via WayPoint, which is a temporary approach target position on the line from $G_{\text {ready }}$ to $P_{\text {ready }}$ and simply set as the position $1 \mathrm{~m}$ away from $G_{\text {ready }}$. This means that if the robot can finish the movement to approach and rotate to the target within the given time, the system considers it feasible.

Further, it tests whether the robot has enough time to extend its arm before arriving to $G_{\text {ready }}$, based on the constraint in section III-B:

CanArmReady $(t)= \begin{cases}\text { true } & (\text { EstimatedArmTime }) \leq t \\ \text { false } & (\text { otherwise })\end{cases}$

where EstimatedArmTime is the maximum moving time for each arm joint of the robot to reach the final position. The handing posture is calculated by the gesture controller which is described later. Thus, if the robot cannot finish extending its arm by the given time, the system does not choose this candidate. 
Finally, the system chooses the target person $i$ who yields the minimum $t$ that satisfies equations (3) and (4), i.e.,

$$
\begin{aligned}
& \text { Target }(i, t, \text { Side })= \\
& \quad \underset{i, t, \text { Side }}{\operatorname{argmin}}(\operatorname{arriveTime}(i, t, \text { Side }) \cdot(\text { isLastTrget }(i)+1)) \\
& \operatorname{arriveTime}(i, t, \text { Side })= \\
& \qquad \begin{array}{l}
\left.t \text { (if CanArrive }\left(t, P_{r}, G_{\text {ready }}(i, t, \text { Side })\right) \& \text { CanArmReady }(t)\right) \\
\infty(\text { otherwise })
\end{array}
\end{aligned}
$$

where the range of $i$ within 0 to number of people, the range of $t$ within 0 to $40 \mathrm{sec}$ for computational economy, and the range of Side is left and right. To prevent oscillation of switching between multiple targets, the target person in the previous round of computation is prioritized in the choice of target in the current round. The function, isLastTarget $(i)$ returns 1 if the target in the previous round is $i$; otherwise it returns 0 . If all candidates are infinity, there is no target pedestrian. The target is updated every $500 \mathrm{msec}$.

As a simple implementation, $E P_{i}(t)$ is estimated by a linear interpolation with the past speed information of the pedestrian $i$ as shown in Figure 5:

$$
E P_{i}(t)=P_{\text {current }}+v^{p} \cdot t
$$

This provided performance that was sufficient for our application, though it could probably be improved using other sophisticated methods, such as described in [20], [21].

\section{Spatial formation controller}

Once a target is selected by the target decider, the spatial formation controller takes responsibility for navigating the robot. It controls the robot to reach WayPoint first, and then follow a line that connects $P_{\text {ready }}$ and $G_{\text {ready }}$ (as shown in Figure 9). Each position is updated every $100 \mathrm{msec}$.

To make the robot reach the $G_{\text {ready }}$ position at appropriate timing, we dynamically adjusted its velocity. We controlled the robot to move with high speed first; and then, in order to adjust arrival timing, it starts to decrease its speed when it gets close to $G_{\text {ready }}$. Fig. 10 illustrates the idea of the control of the speed. In concrete, the forward velocity is controlled by considering the distance and angle to $G_{\text {ready }}$ as follows:

$$
v_{r}=\left\{\begin{array}{l}
v_{\max } \quad\left(\text { if } \frac{\text { RemainingDist }}{\text { RemainingTime }} \cdot \text { TimeToleranceFactor }>v_{\max }\right)(8) \\
\frac{\text { RemainingDist }}{\text { RemainingTime }} \cdot \text { TimeToleranceFactor } \quad \text { (otherwise) }
\end{array}\right.
$$

where $v_{\max }$ is the maximum speed for the robot, RemainingDist is the summation of the distances between $G_{\text {current }}$ to WayPoint and WayPoint to $G_{\text {ready, i.e., D1 }}$, D2 in Fig. 10, RemainingTime is the remaining time to $t_{\text {approachready }}$ by considering current time and the required time for rotating.

Due to the difficulties of finding the timing of receiving, the control of spatial formation lasts when the pedestrian passes the robot. We define $\delta$ as the angle between the pedestrian's moving direction and the vector from the position of the pedestrian to the robot. When $\delta>=90 \mathrm{deg}$, we consider the pedestrian to have passed.

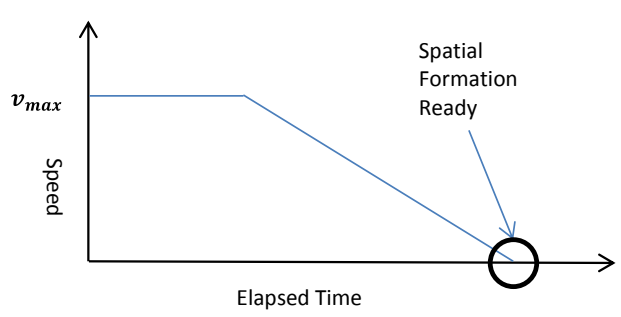

Figure 10. Illustration of changes of robot's speed

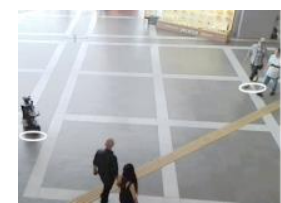

(a)

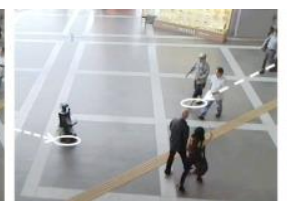

(b)

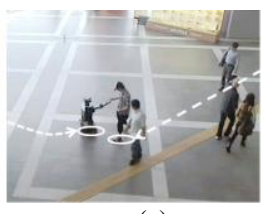

(c)
Figure 11. A pedestrian successfully received while he kept walking

\section{Gesture controller}

This function controls the gaze and arm motion. The gaze behavior is started from the very beginning (as explained in section III-B-(1)). That is, when a target pedestrian has been chosen, the robot's head direction is directed to the pedestrian's head location to create and maintain eye contact. The robot keeps directing its head direction towards the pedestrian until the distributional handing has finished.

For the arm motion, the gesture controller generates a handing motion. As illustrated in figure 6, the height of the robot's hand was calculated by using the target height information as written in equation (2). We had already defined the constraint in section III-B-(2)-(a) to explain that $t_{\text {ready }}$ of the arm is equal to that of approaching behavior. After one distributional handing interaction with a pedestrian, the robot's arm pose is set to the default one as shown in the left image of figure 9. In addition, the robot said "Please (accept a flyer)" to the pedestrians at the time of $t_{\text {armstart }}$.

\section{E. Example}

The Figure 11 shows a scene of successful handing with the method. When the robot found the target, it moved toward the $G_{\text {ready }}$ (Figure 11-a to b), and extended its arm just before its arrival (Figure 11-c). The pedestrians received the flyer without stopping his walk.

\section{EVALUATION}

\section{A. Hypothesis and Prediction}

The proposed method models how humans perform distributional handing. We expect that if this model is designed properly, the robot is able to convey its intention of handing to a target person equally successfully as a human would do.

As an alternative method, we used a method with the knowledge of handing objects to a seated person [22]. This is the current state of the art, as to the best of our knowledge there exists no other method for distributional handing. It is to make the robot approach the target person from front side, and start arm motion as the distance to the receiver got very close. The approaching method and timing for arm motion are different from what we found in this research. The situation that handing 
an object to a seated person is largely different from distributional handing. In distributional handing, the pedestrians are always moving, and they do not know that the giver would handing them the flyer from the beginning. We expect that pedestrians would often fail to understand the robot's intention of handing, and even if they understand its intention, busy people would not wait for the completion of the robot's handing motion since the robot's handing interaction is not performed in a timely fashion. Based on the idea above, we made the prediction as follows:

Prediction 1: With the proposed model, the robot will be more frequently successful in handing flyers (make more subjects receive the flyers) than the robot with an alternative method.

\section{B. Method}

\section{1) Settings}

The evaluation was conducted at a shopping mall where we conducted data collection. The robot was placed in a large hallway $(8 \times 12 \mathrm{~m})$ (Figure 12$)$, which connects an event hall and a train station, and there are restaurants and shops nearby.

\section{2) Comparison and Measurement}

The two methods below were compared.

- Distributional handing (proposed): The robot is controlled by the proposed spatial information controller and the gesture controller described in Section IV.

- $\quad$ Frontal approach and handing nearby: The robot performs handing interaction with a frontal approach and handing basis. In concrete terms, the robot approaches the target person from directly frontal and does not stop until it reaches the secure distance (set to be $1.2 \mathrm{~m}$ ) without considering $G_{\text {ready }}$ constraints. The robot starts the handing motion $0.5 \mathrm{~m}$ away from its target positon. Aside from this difference in the timing of the handing motion and the stop position, other parameters and methods are shared with the proposed method.

We prepared several time slots (morning, midday and afternoon) and counter-balanced the order to carefully ensure that the sets of subjects who are exposed to each condition are equivalent. We measured the performance for each method by calculating the successful ratio.

\section{Results}

\section{1) Verification of prediction}

In the experiment, we conducted 30 trials for each condition. Figure 13 shows the success ratio of the two methods; the ratio of successful handing was $73.3 \%$ (22/30) for proposed method and $33.3 \%$ (10/30) for approach and handing method. A Chi-square test revealed significant differences in the successful ratio of the handing $(\chi 2(1)=8.103, \mathrm{p}<.01, \varphi=$ $0.367)$. Therefore, our prediction 1 was supported.

\section{2) Analysis of scene of interaction}

We analyzed reactions of the targeted person during the robot conducted the handing behavior. All 60 scenes of handing were analyzed. Below are results of coding.

\section{Pedestrians who received the flyer:}

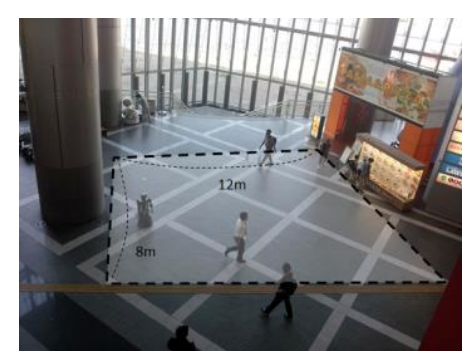

Figure 12. Environment where we conducted the experiment

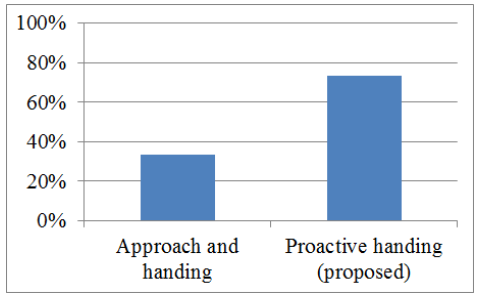

Figure 13. Ratio of successful handing

TABle 2 Classification of PEDEStRians’ ReACtion

\begin{tabular}{|c|c|c|c|}
\hline \multicolumn{2}{|c|}{ Pedestrian's Reaction } & Frontal approach & Distributional \\
\hline \multirow{3}{*}{ Received } & Kept walking & 0 & 4 \\
\hline & Stopped and received & 7 & 17 \\
\hline & Turned back & 3 & 1 \\
\hline \multirow{3}{*}{$\begin{array}{l}\text { Did not } \\
\text { receive }\end{array}$} & Passed by & 0 & 4 \\
\hline & Avoided & 15 & 4 \\
\hline & Stopped and observed & 5 & 0 \\
\hline
\end{tabular}

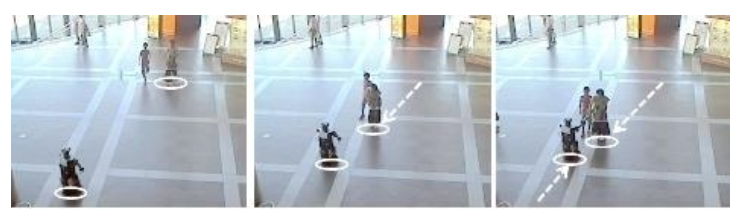

(a)

(b)

(c)

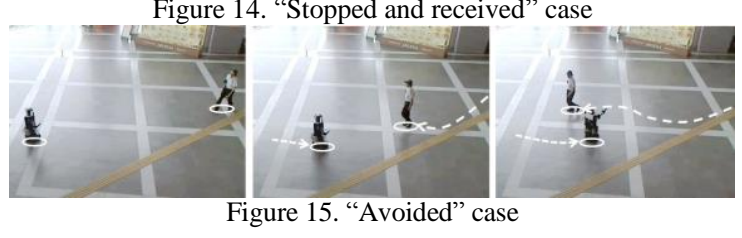

- Kept walking: pedestrians received the flyer during walking, and did not stop their motion (like the case shown in fig. 11).

- Stopped and received: pedestrians slowed down in front of the robot, and stopped to wait the robot approach them and hand the flyer. Finally they received the flyer; they stopped in front of the robot (fig.14-b) and waited (fig.14-c)).

- Turned back: pedestrians once avoided the robot, but they turned back to the robot when it extended arm.

Pedestrians who did not receive the flyer:

- Passed by: pedestrians passed by the robot without changing their moving direction and speed.

- Avoided: pedestrians changed their moving direction to avoid the robot. 
- Stopped and observed: pedestrians stopped and observed the robot; but, when the robot approached and handed the flyer, they did not receive it.

The coding results are shown in the table 2. Cohen's kappa coefficient from the two coders' classifications was 0.869 , indicating that their evaluations were highly consistent. The pedestrians who received the flyer while walking (i.e. "kept walking") are only found in the proposed method. In addition, "passed by" pedestrians were only found in the proposed method. This means that for such people the robot with the proposed model did not disturb their walking. In contrast, there are many "avoided" pedestrians found in the alternative method. Fig. 15 shows an example of "avoided" category in the alternative method, in which the target pedestrian change his course when the robot approached. "Avoided" would indicate that the robot obstructed the pedestrians during handing behavior. We conducted a Chi-square test for the ratio of avoided pedestrians (i.e. "avoided" category) among all pedestrians. The results revealed significant differences between two conditions $(\chi 2(1)=7.702, p<.01, \varphi=0.358)$. It means that the proposed method prevents the robot from obstructing pedestrians and would increase the success ratio.

\section{DISSCUSSION}

In our setting, the robot moved around a large space and approached pedestrians there. However, in other contexts, different motions might be desired. For example, if the robot's task is to distribute pamphlets in a narrow corridor or narrow space (e.g. entrance gate), perhaps the robot should stay at the same location and wait a flow of visitors continuously come through in a line. We believe that our model can be partly used to deal with such interaction. We can compute $G_{\text {ready }}$ position for such potential visitors in a flow, and locate the robot there. The timing computation for timely extend its arm toward the moment visitor arrive to $P_{\text {ready }}$ will be still useful.

The parameters in our model deal with Japanese subjects and our own robots.

\section{CONCLUSION}

We studied distributional handing interactions where a giver meets a pedestrian. From these interactions we created a model of natural human interaction. The model was then implemented into a humanoid robot and tested with an evaluation experiment. We compared the proposed model with a baseline model. The experiment results proved the proposed model is effective.

\section{REFERENCES}

[1] C. C. Kemp, A. Edsinger and E. Torres-Jara, Challenges for Robot Manipulation in Human Environments, IEEE Robotics \& Automation Magazine, vol. 14, pp. 20-29, 2007.

[2] A. Sorokin, D. Berenson, S. S. Srinivasa and M. Hebert, People Helping Robots Helping People: Crowdsourcing for Grasping Novel Objects, IEEE/RSJ Int. Conf. on Intelligent Robots and Systems (IROS2010), pp. 2117-2122, 2010.

[3] M. Ralph and M. A. Moussa, An Integrated System for User-Adaptive Robotic Grasping, IEEE Transactions on Robotics, pp. 1-12, 2010.

[4] S. Kajikawa, T. Okino, K. Ohba and H. Inooka, Motion Planning for Hand-over between Human and Robot, IEEE/RSJ Int. Conf. on Intelligent Robots and Systems (IROS1999), pp. 193-199, 1995.
[5] S. Shibata, K. Tanaka and A. Shimizu, Experimental Analysis of Handing Over, IEEE Int. Workshop on Robot and Human Interactive Communication (RO-MAN1995), pp. 53-58, 1995.

[6] A. Agah and K. Tanie, Human Interaction with a Service Robot: MobileManipulator Handing over an Object to a Human, IEEE Int. Conf. on Robotics and Automation (ICRA1997), pp. 575-580, 1997.

[7] M. Huber, M. Rickert, A. Knoll, T. Brandt and S. Glasauer, HumanRobot Interaction in Handing-over Tasks, IEEE Int. Symposium on Robot and Human Interactive Communication (RO-MAN2008), pp. 107-112, 2008.

[8] M. Cakmak, S. S. Srinivasa, M. K. Lee, S. Kiesler and J. Forlizzi, Using Spatial and Temporal Contrast for Fluent Robot-Human Hand-Overs, ACM/IEEE Int. Conf. on Human-Robot Interaction (HRI 2011), pp. 489-496, 2011.

[9] M. Cakmak, S. S. Srinivasa, M. K. Lee, J. Forlizzi and S. Kiesler, Human Preferences for Robot-Human Hand-over Configurations, IEEE/RSJ Int. Conf. on Intelligent Robots and Systems (IROS2011), pp. 1986-1993, 2011.

[10] E. A. Sisbot, L. F. Marin-Urias, R. Alami and T. Simeon, A Human Aware Mobile Robot Motion Planner, IEEE Transactions on Robotics, vol. 23, pp. 874-883, 2007.

[11] E. A. Sisbot, A. Clodic, R. Alami and M. Ransan, Supervision and Motion Planning for a Mobile Manipulator Interacting with Humans, ACM/IEEE Int. Conf. on Human-Robot Interaction (HRI2008), pp. 327-334, 2008

[12] E. A. Sisbot, R. Ros and R. Alami, Situation Assessment for HumanRobot Interactive Object Manipulation, IEEE Int. Symposium on Robot and Human Interactive Communication (RO-MAN2011), pp. 15-20, 2011.

[13] M. P. Michalowski, S. Sabanovic and R. Simmons, A Spatial Model of Engagement for a Social Robot, IEEE International Workshop on Advanced Motion Control, pp. 762-767, 2006.

[14] N. Bergström, T. Kanda, T. Miyashita, H. Ishiguro and N. Hagita, Modeling of Natural Human-Robot Encounters, IEEE/RSJ Int. Conf. on Intelligent Robots and Systems (IROS2008), pp. 2623-2629, 2008.

[15] K. Dautenhahn, M. L. Walters, S. Woods, K. L. Koay, C. L. Nehaniv, E. A. Sisbot, R. Alami and T. Siméon, How May I Serve You? A Robot Companion Approaching a Seated Person in a Helping Context, ACM/IEEE Int. Conf. on Human-Robot Interaction (HRI2006), pp. 172-179, 2006.

[16] C. Shi, M. Shimada, T. Kanda, H. Ishiguro and N. Hagita, Spatial Formation Model for Initiating Conversation, Robotics: Science and Systems Conference (RSS2011), 2011.

[17] S. Satake, T. Kanda, D. F. Glas, M. Imai, H. Ishiguro and N. Hagita, How to Approach Humans?: Strategies for Social Robots to Initiate Interaction, ACM/IEEE Int. Conf. on Human-Robot Interaction (HRI2009), pp. 109-116, 2009.

[18] S. Glasauer, M. Huber, P. Basili, A. Knoll and T. Brandt, Interacting in time and space: Investigating human-human and human-robot joint action, IEEE Ro-Man 2010, pp 252-257, 2010

[19] T. Kanda, H. Ishiguro, M. Imai and T. Ono, Development and Evaluation of Interactive Humanoid Robots, Proceedings of the IEEE, vol. 92, pp. 1839-1850, 2004.

[20] M. Bennewitz, W. Burgard, G. Cielniak and S. Thrun, Learning Motion Patterns of People for Compliant Robot Motion, Internationl Journal of Robotics Research, vol. 24, pp. 31-48, 2005.

[21] T. Kanda, D. F. Glas, M. Shiomi and N. Hagita, Abstracting People's Trajectories for Social Robots to Proactively Approach Customers, IEEE Transactions on Robotics, vol. 25, pp. 1382-1396, 2009.

[22] K.L. Koay, E.A. Sisbot, D.S. Syrdal, M.L. Walters, K. Dautenhahn and R.Alami, Exploratory Study of a Robot Approaching a Person in the Context of Handing Over an Object, In Proceedings of AAAI SpringSymposium2007: SS07, Multidisciplinary Collaborationfor Socially Assistive Robotics, AAAI Technical Report, Stanford University, Palo Alto, Ca, USA. pp. 1824 AAAI Press 